\title{
KAIRÓS Y VULNERABILIDAD DE LAS PERSONAS AFECTADAS POR ENFERMEDADES RARAS: UN ANÁLISIS SEMIÓTICO Y HERMENÉUTICO
}

\author{
Kairós and the Vulnerability of People Affected by Rare Diseases: \\ A Semiotic and Hermeneutic Analysis
}

\author{
Juan R. Coca, Universidad de Valladolid, España \\ Correo electrónico: juanr.coca@uva.es \\ Juan A. Roche-Cárcel, Universidad de Alicante, España \\ Correo electrónico: ja.roche@ua.es
}

Recibido: $15 / 05 / 2021$

Aceptado: $15 / 11 / 2021$

Publicado: 07/01/2022

\begin{abstract}
Resumen. En este artículo se realiza un análisis teórico de los condicionantes vitales que tienen las personas afectadas por enfermedades raras y sus familias. Esta investigación se estructura desde una perspectiva semiótica y hermenéutica para poder comprender mejor su realidad social. En ella hemos detectado la inexistencia del Kairós y el imperio del chronos. Este hecho es fruto de la estructuración del sistema social y de la limitación de opciones para estas personas. Ante esta realidad, se plantea la posibilidad de estructurar una sociedad donde se produzca una reducción kairológica y un fomento de la fraternidad, entendida como alternativa temporal.
\end{abstract}

Palabras clave: tiempo social, salud, enfermedades raras, teoría social.

Abstract. In this paper, a theoretical analysis of the vital conditioning factors that people affected by rare diseases and their families have is carried out. This research is structured from a semiotic and hermeneutic perspective in order to better understand their social reality. In it we have detected the inexistence of the Kairos and the empire of the chronos. This fact is the result of the structuring of the social system and the limitation of options for these people. Faced with this reality, the possibility of structuring a society where there is a kairological reduction and a promotion of fraternity, understood as a temporary alternative, is raised. Keywords: social time, health, rare diseases, social theory.

Cómo citar: Roca, J. R. y Roche-Cárcel, J. A. (2022). Kairós y vulnerabilidad de las personas afectadas por enfermedades raras: un análisis semiótico y hermenéutico. EN-CLAVES del pensamiento, 0(31), e494. doi: https://doi.org/10.46530/ecdp.v0i31.494 


\section{Introducción}

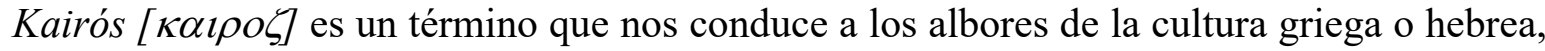
que contiene un significado realmente complejo y repleto de matices. Kjeldsen ${ }^{1}$ indicó que los investigadores, que se han ocupado de conocer más sobre este término, se han centrado en dos aspectos fundamentales de su significado. Por un lado, en la oportunidad de hacer algo en un determinado momento, estando relacionado — en cierto modo - con la medida

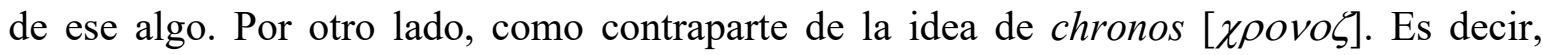
mientras que la idea de chronos se vincula con un flujo permanente y constate, Kairós será una puntualización, un instante en el chronos, pero abierto a la posibilidad.

Muga $^{2}$ mostró que existen diferencias entre el concepto de chronos hebreo (estudiado en la concepción antropológica de Pablo de Tarso) y el chronos griego. En el primero, la dimensión temporal se encuentra acentuada, mientras que en el chronos griego hay un triple sentido relacionado con lo local, lo objetivo y lo temporal. Dicha distinción cultural también la vemos en el Kairós, aunque con matices. En este caso, la diferencia fundamental se encuentra, de un modo simplificado, en la manera en que el Kairós se relaciona con la existencia. En la concepción griega, este tiempo exige decisión y acción al ser humano. Hasta el punto de que negarse a tal agencia sería llevar a cabo una renuncia a la existencia. Dicho de otro modo, en la Grecia clásica se interpretaba esa oportunidad, ese Kairós, como una opción tanto positiva (esa obligación de ser quién eres), como negativa (ruptura con la existencia). En cambio, el Kairós hebreo no presenta ese carácter dramático e incluso fatalista presente en el mundo griego. ${ }^{3}$ Este tiempo, para los judíos, sería un instante para la esperanza, por lo que la acción humana implicaría la capacidad de desarrollar toda la potencialidad humana.

Con independencia de los matices establecidos en cada contexto cultural, podemos hablar de la existencia de dos elementos que tienen interés para nuestro trabajo: la apertura a la posibilidad y, en segundo lugar, el condicionante del contexto. En una visión

\footnotetext{
${ }^{1}$ Jens E. Kjeldsen, "Reconceptualizing Kairos", en Eyjólfur Kjalar Emilsson, Anastasia Maravela y Mathilde Skoie (eds), Paradeigmata: studies in honour of Øivind Andersen (Athens: The Norwegian Institute at Athens, 2014), 249-258.

${ }^{2}$ Jesús Muga, El tiempo hebreo. Referentes antropológicos (Madrid: Fundación Emmanuel Mounier, 2002), 98.

${ }^{3}$ Ibid., 101.
} 
más actual del término, Niles et al. ${ }^{4}$ afirman que el Kairós, tiene relación con el ámbito de la experiencia y por esta razón puede vincularse con elementos narrativos y con los sentimientos. En este sentido, incluso, podríamos afirmar que el Kairós estaría vinculado con la interpretación del mundo, de lo que nos sucede (hermenéutica) y, por ende, con la significación que se le otorga al mundo (semiótica). Vemos, entonces, que el Kairós mantendría una estrecha relación entre la subjetividad en su relación con el contexto y con la posibilidad de concebir el momento temporal como oportunidad.

En este sentido, el Kairós, en el ámbito de la salud y del cuidado (que es lo que nos ocupa ahora), se podría entrelazar con el cambio gradual, con las interacciones, con los momentos, con lo íntimo, con las transiciones y con las transformaciones. ${ }^{5} \mathrm{Al}$ fin y al cabo, la enfermedad puede constituirse como un reto personal y familiar, aunque ello depende del tipo de enfermedad del que estemos hablando.

Estamos, por lo tanto, ante un elemento hermenéutico de gran importancia. El tiempo en su doble dimensión (chronos y Kairós) será analógico al transitar entre el mundo de lo objetivo y de lo subjetivo. Esta idea de un tiempo analógico necesita de una corporalización del mismo, puesto que, sin tal fenómeno de mediación, sería difícil la conceptualización del tiempo. Dicho de otro modo, los procesos naturales son percibidos gracias a nuestro cuerpo y a los efectos que los ciclos vitales generan sobre nosotros. Por lo tanto, a través de esta corporalización del tiempo, se ha podido conformar las estructuras temporales de las que estamos hablando. Por esta razón podríamos afirmar que las dos dimensiones del tiempo estarían asentadas en una hermenéutica biosocial del mundo, donde la vida se percibe corporalmente en función de una interpretación cultural de la misma.

A todo esto, habría que añadir otro aspecto. Es necesario ser conscientes de la existencia del giro afectivo del que ha hablado Crespo $^{6}$ en clara relación con el giro kantiano (del objeto al sujeto) y con el giro lingüístico (de la conciencia al lenguaje).

\footnotetext{
${ }^{4}$ Paulomi Mimi Niles, Saraswathi Vedam, Amy Witkoski Stimpfel y Allison Squires, "Kairos Care in a Chronos World: Midwifery Care as Model of Resistance and Accountability in Public Health Settings", Birth. Issues in Perinatal Care, núm. 00 (2021): 1-13. https://doi.org/10.1111/birt.12565.

${ }^{5}$ Ibidem.

${ }^{6}$ Eduardo Crespo, "Un enfoque social sobre las emociones", en José Luis Álvaro (coord.), La interacción social. Escritos en homenaje a José Ramón Torregrosa (Madrid: CIS, 2018), 167.
} 
Dicho giro afectivo se asienta sobre la idea de que el orden social y su mecanismo de institucionalización terminan, de un modo u otro, estableciendo un correlato corporal. Esta idea tiene un enorme interés, puesto que implica la aceptación de que nuestro entorno (lo que podría denominar como el nicho social o umwelt de acuerdo con la biosemiótica) como elemento conformador y resignificador de nuestro cuerpo. En función de esto, podemos afirmar que la manera en que estructuramos el tiempo social también tiene gran relevancia por su consiguiente efecto en la corporalidad humana.

En este sentido, el objetivo de nuestro trabajo será analizar la concepción del Kairós en las personas afectadas por enfermedades raras y en sus familias. Es decir, en personas que ven alterada, de un modo u otro, su corporalidad.

\section{El self en el contexto de las enfermedades raras}

Las enfermedades raras o poco frecuentes son un conjunto de patologías que se definen, fundamentalmente, por afectar a un número reducido de personas. Por ello, dependiendo del caso, el nivel de prevalencia de estas enfermedades será realmente bajo. Actualmente, podemos indicar que existen entre 6.000 y 8.000 enfermedades raras. Este rango es amplio puesto que, de acuerdo con Berman, ${ }^{7}$ cabe la posibilidad de que el actual número esté infravalorado. Este hecho es debido a que existe un profundo desconocimiento sobre este tipo de patologías, lo que, como veremos, se convertirá en uno de los principales determinantes sociales y personales de los afectados y sus familias.

El concepto de Enfermedad Rara no es homogéneo. En función del lugar donde nos encontremos, se establece un dato epidemiológico que será el que establezca el apelativo de "raro". Por ejemplo, en el caso europeo esta designación hace referencia a aquellas patologías que afectan a menos de 5 de cada 10.000 personas. En cambio, en los Estados Unidos de América, dicha cifra asciende a 7,5 de cada 10.000. Así mismo, regiones como Australia o Japón muestran una definición conceptual más restringida y reducen el término a 4 personas por cada 10.000 habitantes. Si incluimos en esta conceptualización también las enfermedades denominadas como ultra-raras, el rango de

\footnotetext{
7 John Berman, Enfermedades raras y medicamentos huérfanos. Claves para entender y tratar las enfermedades comunes (Barcelona: Elsevier, 2015), 3.
} 
prevalencia oscila entre 1 caso por cada 1.000.000 de personas, hasta los 15 casos por 10.000 personas, que se llegó a utilizar en una definición de una organización general de pacientes en China. ${ }^{8}$

Bajo el término 'paraguas' de raras se engloban patologías en su mayor parte genéticas, tendentes a la cronicidad y muchas veces muy limitantes (ya sea en el área sensorial, motora o intelectual), que pueden llevar aparejadas alteraciones severas en el lenguaje y la cognición y un progresivo deterioro en la autonomía y la calidad de vida. Por todo lo antedicho, puede decirse que las enfermedades raras, como fenómeno colectivo, conforman un grupo social más o menos delimitado, el cual comparte realidades familiares, personales, laborales y socioeducativas definidas por su orfandad social, su complejidad y su permanencia en el tiempo.

Entre esa cantidad tan grande de enfermedades raras, nos encontramos con afecciones muy complejas y graves, y también con afecciones que permiten a las personas realizar una vida sin graves limitaciones. Las personas con patologías muy complejas, cuyo efecto es mayor, pueden terminar yendo, de manera constante, a los hospitales y a los centros de salud. Ello hace que su vida, sus relaciones, sus vivencias personales, en definitiva, su mundo-de-la-vida se vea sumamente limitado. ${ }^{9}$ Ello es debido a que el mundo intersubjetivo se ve reducido por los condicionantes biosociales de las personas afectadas y de sus familias. Estos condicionantes pueden estar relacionados, en función de la enfermedad de la que estemos hablando, por las discapacidades visuales, corporales, mentales, auditivas, etc., que se puedan generar. Para aclarar brevemente a qué nos referimos cuando hablamos del mundo-de-la-vida recurriremos a Habermas ${ }^{10}$ quien logra explicar muy bien este concepto. Este autor, indica que está relacionado con la acción comunicativa debido a que es un fenómeno de configuración de sentido de la realidad circundante y ésta necesita de cierto consenso con los demás. Además, indica también

\footnotetext{
${ }^{8}$ Trevor Richter, Sandra Nestler-Parr, Robert Babela, Zeba M. Khan, Theresa Tesoro, Elizabeth Molsen, Dyfrig Hughes, et al. "Rare Disease Terminology and Definitions - A Systematic Global Review: Report of the ISPOR Rare Disease Special Interest Group", Value in Health 6, núm. 18 (2015): 906-914. https://doi.org/10.1016/j.jval.2015.05.008.

9 Patrice F. Band, "Vignette: Autoimmune Polyendocrine Syndrome Type I (APS 1)", en Rajeev K. Bali, Lodewijs Bos, Michael Christopher Gibbons y Simon Ibell (eds.), Rare Diseases in the Age of Health 2.0 (Berlin/Heidelberg: Springer-Verlag, 2014): 21-25. https://dx.doi.org/10.1007/978-3-642-38643-5_2.

${ }^{10}$ Jünger Habermas, Teoría de la acción comunicativa (Madrid: Trotta, 2018).
} 
que el sistema social interviene, de manera constante, en este mundo-de-la-vida. En este sentido, Habermas ${ }^{11}$ nos indica, de manera estructuralista, que este sentido del mundo se establece de acuerdo a elementos subjetivos (personalidad), normas colectivas (sociedad) y patrones de interpretación comunes (cultura), todos ellos configurados de manera concéntrica. De ahí que, a medida que nos alejamos del centro de dicho mundo, aumenta la distancia espacio-temporal y también la distancia social.

Pues bien, las limitaciones relacionadas con las enfermedades raras reducen las posibilidades de las personas afectadas por generar diferentes tipos de discapacidades o condicionantes biomédicos. A su vez, afecta a las familias ya que éstas tienen que atender y cuidar de las personas enfermas, restringiendo también sus posibilidades sociales. Por otro lado, las manifestaciones que producen muchas de estas patologías también se han visto afectadas por procesos de rechazo y de exclusión social; en buena medida fruto del desconocimiento ajeno. Todo ello, por lo tanto, limita el mundo-de-la-vida, puesto que la estructura concéntrica que se genera en las vidas de estas personas y de sus familias no es tan amplia como el de las personas sin estas afecciones. En línea con esto, si se reduce este espacio, también se limitará el entorno de sentido, la posibilidad sociosemiótica y sociohermenéutica de estas personas.

En este sentido, la aproximación general que estableció Erving Goffman en su obra Estigma, si bien no permite comprender adecuadamente la realidad de estas personas, sí presenta elementos que se ajustan a las vivencias de ellas. Cuando Goffman habla del enfermo indica que "si maneja en forma correcta su estatus de enfermo puede apartarse de los estándares de funcionamiento, sin que esto sea tomado como un reproche hacia él o hacia su relación con el grupo". ${ }^{12}$ De acuerdo con investigaciones de carácter social, ${ }^{13}$ las personas afectadas por enfermedades raras se sienten excluidas y, en algunos casos, llegan a aislarse notablemente de la sociedad. Ello nos lleva a pensar que la

\footnotetext{
${ }^{11}$ Ibidem.

${ }^{12}$ Erving Goffman, Estigma. La identidad deteriorada (Buenos Aires: Amorrortu, 2010), 176.

13 Juan R. Coca (coord.), Enfermedades raras. Contribuciones a la investigación social y biomédica (Soria: CEASGA, 2019); Agustín Huete y Eduardo Díaz, Estudio sobre situación de Necesidades Sociosanitarias de las personas con Enfermedades Raras en España. Estudio ENSERio. (Madrid: FEDER, 2009); Charlotte von der Lippe, Plata S. Diesen y Kristin B. Feragen, "Living with a Rare Disorder: A Systematic Review of the Qualitative Literature", Molecular Genetics \& Genomic Medicine, 6 núm. 5 (2017): 758-773. https://dx.doi.org/10.1002/mgg3.315.
} 
perspectiva de Goffman ${ }^{14}$ no se ajusta a una exclusión tan acusada como la que viven estas personas.

Por otro lado, las enfermedades raras mantienen cierta relación con las crónicas. Ello es debido a que las primeras se manifiestan (muchas de ellas) como afecciones de larga duración e, incluso, llegan a tener una progresión lenta. Este hecho hace que los logros biomédicos y psicosociales que puedan alcanzar las personas afectadas sean paulatinos. Ahora bien, esto también puede traer consigo ciertos retrocesos. Estamos hablando, por lo tanto, de la existencia de unos condicionantes biosociales que afectan a la configuración del self de las personas que tienen la enfermedad y de sus familias. Dicho de otro modo, los condicionantes de los que hemos hablado (y, por supuesto, hay muchos más) obligan a los afectados a desarrollar una hermenéutica, en ocasiones, limitada del mundo-de-la-vida. Ello no quiere decir que sus capacidades se vean mermadas, sino que los procesos de exclusión y de reducción de su mundo-de-la-vida generan también limitaciones en su capacidad para interpretar el mundo.

En este punto es importante recuperar algunas ideas expuestas por Bericat. ${ }^{15}$ En este estudio sociológico, se indica que las emociones individuales son una pauta emocional que deriva, a su vez, de la interacción entre el self y lo social (especialmente los otros). Evidentemente esta pauta emocional está referida al concepto de sentido. Pero no hablamos de un sentido metafísico, sino de una significación relacional. Al respecto, podemos usar las palabras de Lévinas para asentar, todavía más esta idea.

La legitimidad de ser, pregunta disimulada en la más banal y más lancinante: en la que investiga el "sentido de la vida" sin contentarse con las finalidades biológicas, psicológicas o sociológicas de la existencia. Pregunta a través de la cual significa originariamente la misma significatividad del ser; sentido que no se define simplemente por el formalismo de la estructura lógica de la referencia, que va de un significante cualquiera a su significado; o, más exactamente, sentido en el que la referencia emerge en toda la concreción del uno-para-el-otro de la fraternidad humana. ${ }^{16}$

Dicho de otro modo, la construcción del self (mi propio yo) se establece en relación con otras personas. Ahora bien, siempre y cuando el otro me permita ser. En este sentido, toda

\footnotetext{
${ }^{14}$ Ibidem.

${ }^{15}$ Eduardo Bericat, Excluidos de la felicidad. La estratificación social del bienestar emocional en España (Madrid: CIS, 2018), 291 y ss.

${ }^{16}$ Emmanuel Lévinas, Fuera del sujeto (Madrid: Caparrós Editores, 1997).
} 
persona, por el mero hecho de serlo, tiene legitimidad ontológica para ser. La cuestión aquí no es esa. De lo que estamos hablando es de la legitimidad sociológica de ser, que es diferente de la ontológica. En este sentido, las pretensiones de homogeneización social de las que habla Emma León ${ }^{17}$ implica una reducción de dicha legitimidad social, ya que a determinadas personas se les reduce el mundo social. Esto responde a un ejercicio de dominación del sentido que le otorgan al mundo de la vida unos grupos sociales, frente al sentido de otros grupos sociales. Esta visión está asentada en la hermenéutica restrictiva de un mundo donde solamente tienen cabida aquellos con poder y no otros. En línea con esto, Beuchot, ${ }^{18}$ en repetidas ocasiones ha defendido que el ser humano es un análogo, un mestizo. Un organismo conformado en la diversidad y en clara relación con los demás. Por esta razón, la hermenéutica analógica que promueve y defiende Beuchot es una interpretación con una clara vinculación relacional y social que nos ayuda a conformar un mundo más dialogante.

Ahora bien, es evidente que no podemos dejar a un lado la parte ontológica, puesto que, como indica Mauricio Beuchot, ${ }^{19}$ el ser humano comprende, interpreta y vive el mundo que le rodea en sí mismo. Esto es, en función de cómo nos situemos, o nos sitúen en el mundo, así lo interpretaremos. Beuchot, precisamente, indica que si nos reconocemos a nosotros mismos como microcosmos (como una especie de análogo del ser, del mundo entero - diremos nosotros- en pequeño), podremos también reconocer este carácter en los otros, lo que abre la puerta al reconocimiento de las semejanzas y diferencias del otro $^{20}$. De esta idea se puede colegir que, si los otros no otorgan la legitimidad para ser, limitando el acceso a la salud, a la educación, o estableciendo una sociedad en la que las personas con enfermedades raras se encuentran marginalizadas, entonces los afectados configurarán su mundo-de-la-vida desde una perspectiva de sentido asentada en la agresión, el desprecio, la marginalización, etc. Esto produce, como

\footnotetext{
${ }^{17}$ Emma León, Sentido ajeno: competencias ontológicas y otredad (Barcelona: Anthropos, 2005).

${ }_{18}$ Mauricio Beuchot, Las caras del símbolo: el ícono y el ídolo (Madrid: Caparrós editores, 1999); Mauricio Beuchot, Tratado de hermenéutica analógica. Hacia un nuevo modelo de interpretación (México: UNAM, 2015); Mauricio Beuchot, Hechos e interpretaciones. Hacia una hermenéutica analógica (México: FCE, 2016).

${ }^{19}$ Mauricio Beuchot, Las caras del símbolo, 51 y ss.

${ }^{20}$ Ibidem.
} 
mostró Bericat, ${ }^{21}$ infelicidad, aunque también va a reducir sus capacidades como agente social. Por esta razón, es fundamental defender los procesos y mecanismos sociales y políticos que garanticen las posibilidades de todas las personas para que puedan desarrollarse como agentes sociales.

\section{Estructura, vulnerabilidad y agencia social}

El sistema social, desde una perspectiva estructuralista, puede concebirse como un andamio [scaffold]. La metáfora del andamio nos permite visualizar con facilidad la explicación que vamos a dar a continuación. Unas personas estarán en la parte superior de la estructura y otras en la inferior. Las capas más altas de la estructura lograrán contemplar más atinadamente su espacio y podrán tomar mejores decisiones. Las capas más bajas, todo lo contrario. Por ello, una sociedad que tenga interiorizada en sí misma los valores de la equidad, de la libertad y de la fraternidad, desarrollará políticas que permitan a sus ciudadanos tener la oportunidad de ascender en la estructura social [scaffolding].

El concepto de scaffolding ha sido estudiado, fundamentalmente, por estructuralistas y por semióticos. A nuestro juicio, sería interesante realizar un análisis desde una perspectiva sociohermenéutica para ayudar a comprender mejor este proceso. Bratman $^{22}$ ha mostrado que la capacidad de agencia (de realizar actividades) de las personas puede ser compartida, siempre y cuando estemos hablando desde una perspectiva social. Pues bien, en este sentido, la agencia será compartida cuando exista una estructura de intenciones compartidas interpersonalmente, afirma Bratman. Al fin y al cabo, la sociedad se estructura en función de un conjunto de normas que guían el comportamiento individual de las personas, las cuales van a planificar su agencia individual en función de tales construcciones normativas. Estas normas no tienen que ser legales, pues hablamos también de las normas de comportamiento, de las normas de educación y de otras. De ahí que la intencionalidad de un determinado grupo social, que tiene poder suficiente para establecer una norma o hacer que determinados

\footnotetext{
${ }^{21}$ Ibidem.

${ }^{22}$ Michael E. Bratman, Structures of Agency: Essays (New York: Oxford University Press, 2007); Michael E. Bratman, "Shared Agency", en Chysostomos Mantzavinos (ed.), Philosophy of the Social Sciences. Philosophical Theory and Scientific Practice (Cambridge: Cambridge University Press, 2009).
} 
comportamientos permanezcan, puede llegar a limitar o promover las opciones de otro grupo (tal y como hemos visto antes cuando hablamos del proceso de legitimación social). Esto ha sido denominado por Bratman ${ }^{23}$ como intencionalidad coparticipada o entrelazamiento. Por ello, es importante que existan elementos externos que garanticen la agencia de todos los colectivos.

Por otro lado, Haslam et al. ${ }^{24}$ realizaron un meta-análisis donde comprobaron que el apoyo social y la integración social son ambos elementos de gran relevancia para mantener la salud de las personas y evitar la mortalidad. Además, detectaron que su importancia es comparable a comportamientos de riesgo tales como el tabaquismo, el consumo de alcohol, etc. Por esta razón, estos autores defienden la importancia de los factores sociales como condicionantes destacados para el fomento o el mantenimiento de la salud. Dicho de otro modo, el fenómeno de scaffolding en la estructura social tiene gran relevancia para el mantenimiento de un mundo-de-la-vida saludable. En este sentido, las enfermedades raras nos están planteando un gran reto sociohermenéutico y sociosemiótico. Dicho reto está asentado en la conformación de la estructura social basada en la legitimidad conformada por unos pocos (oligarquías, democráticas o no) o por la diversidad de grupos sociales análogos (fratiarquía).

Por otra parte, hablar de la agencia implica hablar de aspectos ontológicos de la "humanidad" y de la "socialidad". Margaret S. Archer, ${ }^{25}$ en su obra Being Human, estudia este aspecto desde una perspectiva intermedia entre la idea del "Hombre moderno" y del “Ser social”. El primero, considera ella, parte de la afirmación de que la sociedad no contribuye a nuestra propia configuración y, por ello, reafirma una concepción individualista del ser humano. El segundo, en cambio, se establece en base a todo lo contrario; la sociedad contribuye completa y totalmente en nuestra propia configuración personal y apuesta por una visión colectivista de la humanidad. Archer ${ }^{26}$ aboga, entonces,

\footnotetext{
${ }^{23}$ Ibidem.

${ }^{24}$ Alexander Haslam, Charlotte McMahon, Tegan Cruwys, Catherine Haslam, Jolanda Jetten y Niklas Steffens, "Social Cure, What Social Cure? The Propensity to Underestimate the Importance of Social Factors for Health”, Social Science \& Medicine, núm. 198 (2018): 14-21. https://doi.org/10.1016/j.socscimed.2017.12 .020 .

${ }^{25}$ Margaret S. Archer, Being Human: The Problem of Agency (Cambridge: Cambridge University Press, 2009), 51 y ss.

${ }^{26}$ Ibid., 279.
} 
por una visión analógica (en el sentido beuchotiano) y estratificada del "sujeto", donde se produce una "doble morfogénesis" de su agencia. En ella la capacidad de agencia (de acción) implica relación con la estructura y con la cultura (con lo institucional y colectivo), al tiempo que supone una elaboración de uno mismo en el proceso (desarrollo del self). Esta idea la pudimos ver también en George Mead, ${ }^{27}$ quien indicó que el self está relacionado con los otros a través de procesos comunicativos y al tiempo que incorpora para sí elementos establecidos en la comunidad. Vemos entonces que existen concomitancias en la visión de Archer y Mead. Ahora bien, todo lo que hemos dicho es importante, pero necesita ser comprendido gracias al proceso de scaffolding del que hemos hablado antes. Este fenómeno social complementa al desarrollo del self a través de la conformación de la agencia, la cual también entra a formar parte de los procesos sociales genéricos de los que habla Scott Grills ${ }^{28}$ y que, junto con el desarrollo del self y del scaffolding, tienen gran importancia para comprender el mundo-de-la-vida de los grupos sociales más vulnerables.

Los tres conceptos de los que hablamos se entrelazan en esta concepción genérica de lo social. Dicho de otro modo, serán dos elementos importantes de mundo transsubjetivos $^{29}$ que nos permiten comprender la estructura ontológica de lo social. Evidentemente no son los únicos elementos existentes, pero sí son relevantes para esta investigación. Por lo tanto, a medida que el sujeto asciende entre los diferentes estratos (scaffolding) emergerán distintas propiedades y poderes en cada nivel. Margaret Archer, en diferentes trabajos, ha diferenciado entre cuatro estratos: ${ }^{30}$ el self, la persona, el agente y el actor. A cada nivel le corresponderá unas características diferenciales, de tal manera que el nivel del self estará circunscrito al ámbito privado y tendrá un tipo de realización individual. El nivel personal, por ejemplo, se corresponde con un tipo de agencia

\footnotetext{
${ }^{27}$ George Herbert Mead, Mind Self \& Society. The Definitive Edition. Edición de Charles W. Morris, edición anotada de Daniel R. Huebner y Hans Joas (Chicago, London: The University of Chicago Press, 2015).

28 Scott Grills, "Understanding Everyday Life: Generic Social Processes and the Pursuit of Transcontextuality", Symbolic Interaction 4, núm 43 (2019): 615-636.

29 Juan R. Coca y Susana Gómez-Redondo, "Multidimensional Sociohermeneutics on Social Studies: A Pragmatic and Integrative Perspective", en VV.AA., Approaches to the Concept of Trans-Subjectivity (Soria: CEASGA, 2020), 125-152.

${ }^{30}$ Margaret S. Archer, Realist Social Theory: The Morphogenetic Approach (Cambridge: Cambridge University Press, 1995); Margaret S. Archer, Culture and Agency: The Place of Culture in Social Theory (Cambridge: Cambridge University Press, 1996); Archer, Being Human, 2009.
} 
primaria, con un carácter privado y una realización social o colectiva. El nivel de agente, por su parte, supone un nivel de realización colectiva, un carácter eminentemente público donde la persona se agrupa. Estos grupos sociales contribuirán al proceso de reproducción y cambio de la estructura social. En este sentido, Archer ${ }^{31}$ llega incluso a considerar que estos agentes son corporativos. En último lugar nos encontramos con el actor, el cual tiene un carácter triple, puesto que está conformado por personas y por grupos sociales (por lo que tiene también carácter público). Pero, además, estas personas y grupos sociales están estructuradas en organizaciones $y$, por ende, cumplen un papel organizacional que no tienen los anteriores. De este modo, Archer ${ }^{32}$ estructura un continuum a través del cual se establece una interrelación entre diferentes niveles de agencia y de actividad humana bien sea individual o colectiva.

Teniendo en cuenta esta idea de la intencionalidad entrelazada, y siguiendo el modelo morfogenético de Archer, se podría afirmar que las enfermedades raras implican una serie de limitaciones biológicas y médicas que, en buena parte de ellas, generan dificultades para que las personas afectadas puedan adaptarse a la estructura social establecida, así como a sus normas. En este sentido, autores como $\operatorname{Mead}^{33}$ y Habermas ${ }^{34}$ han mostrado, de un modo u otro, que la incorporación de las personas a la estructura y a las normas de la sociedad, está asentada en un proceso de carácter comunicativo. Así, las limitaciones biomédicas muestran cierto correlato social en el que se producen limitaciones también en la capacidad de agencia y, por ende, de scaffolding. Ahora bien, como hemos indicado anteriormente, estas limitaciones sociales están relacionadas con la estructuración social y con la lógica homogeneizadora.

En todo este proceso, cada persona pueda interactuar dentro del sentido colectivo que se ha sido establecido socialmente. Dicho sentido, se refiere a la comunicación verbal y también a la no verbal, la cual no es simplemente la relacionada con la gestualidad, sino que hace referencia al intercambio de información en su sentido más amplio (memética, biosemiótica, etc.). Pongamos un ejemplo, si una persona con movilidad reducida no es capaz de utilizar una acera, entonces simbólicamente este constructo social (la acera)

\footnotetext{
${ }^{31}$ Archer, Being Human, 2009.

${ }^{32}$ Ibidem.

${ }^{33}$ Ibidem.

${ }^{34}$ Ibidem.
} 
pierde parte del sentido establecido previamente. Ello es debido a que esta persona no podrá transitar por ella y, por lo tanto, su 'acera' será, por ejemplo, la propia carretera. En este sentido, parece obvio que el sentido de los elementos sociales se ve alterado por las limitaciones que se establecen socialmente.

Este cambio o modificación en la significación no es algo que tenga que ser entendido como negativo. Habitualmente las personas solemos modificar a los objetos y a los sentidos para los que, en principio, son construidos otorgándoles una nueva significación en función de la interpretación que hagamos de ellos. En este sentido, una mesa la utilizamos como silla, un lapicero como rascador, etc. El problema fundamental estaría, entonces, en si este proceso de interpretación se asienta en un condicionante coercitivo previo. El cual estaría establecido en función del cambio de sentido que un determinado grupo social obliga a realizar a otro. En este proceso el self de la persona afectada por una enfermedad rara estaría demasiado condicionado por los demás, por las limitaciones en su capacidad de agencia y por las reducciones en su capacidad para moverse por la estructura social. No permitiéndose, entonces, un fenómeno de desarrollo personal autónomo (siempre y cuando la enfermedad en cuestión permita tal desarrollo).

Lo que se ha expuesto hace que las personas con enfermedades raras y sus familias tengan limitada su capacidad de alcanzar los niveles superiores de la estructura social. Por consiguiente, habría que analizar si, precisamente, la estructuración social existente (y configurada por grupos sociales sin estas afecciones) es el que limita dicho scaffolding. En este sentido, sabemos que las patologías crónicas (una buena parte de ellas son enfermedades raras) presentan un problema de inadaptación psicosocial a la estructura existente. Este colectivo presenta altos niveles de absentismo laboral producido por la constante visita al médico. ${ }^{35}$ Así mismo, y unido a lo anterior, este colectivo también presenta cierto grado de aislamiento social. ${ }^{36}$ Recuérdese que el colectivo de personas con enfermedades raras y sus familias ven mermada su posibilidad de tener tiempo para su ocio o para actividades libres que les permita desconectar de la enfermedad o de la discapacidad. Además, como ya hemos indicado, existe un gran

\footnotetext{
${ }^{35}$ María Dueñas, Begoña Ojeda, Alejandro Salazar, Juan Antonio Mico e Inmaculada Failde, "A Review of Chronic Pain Impact on Patients, Their Social Environment and the Health Care System", Journal of Pain Research, núm. 9 (2016): 457-467. https://doi.org/10.2147/JPR.S105892.

${ }^{36}$ Ibidem.
} 
desconocimiento alrededor de las enfermedades raras. Ello hace que las personas afectadas vivan con altos niveles de inseguridad e incertidumbre.

\section{A modo de conclusión: La ruptura del imperio del chronos por la communitas}

El concepto de Kairós tiene, como hemos visto el carácter de oportunidad. Ahora bien, es un carácter que, tal y como se ha visto en algunos trabajos relacionados con la salud, parece ser interpretado en sentido positivo: es un instante para ser aprovechado y tener, así, la oportunidad de poner en marcha esa agencia. Evidentemente, esta oportunidad tiene que ser percibida y, por eso, también está vinculado con aspectos subjetivos. Dicho de otro modo, el Kairós necesita dos factores: el desarrollo del self y la posibilidad de agencia.

El desarrollo del self es imprescindible para poder tomar opciones, decidir, realizar actividades, etc. En este sentido, las capacidades internas son un elemento vital para poder conformar un mundo-de-la-vida amplio y desarrollado. Por esta razón, los efectos emocionales que traen consigo las enfermedades raras, limitan tal desarrollo. A su vez, esto también se termina configurando como un factor determinante de la posibilidad de percibir el mundo o un suceso como un Kairós.

En segundo lugar, estas afecciones también afectan a la capacidad de agencia y a las posibilidades de scaffolding social. La estructura social está tan determinada por la mayoría que sitúa en el margen de la estructura a estos y otros colectivos. Nuevamente, esto limita las opciones vitales y la posible percepción de un momento vital como oportunidad (Kairós).

Abundando en estas ideas, Fredric Jameson ${ }^{37}$ afirmó, entonces, que era necesaria una ruptura kairológica para que fuese posible la utopía y la transformación del mundo. Este autor, precisamente, considera que la transformación social y laboral necesita de esta ruptura para evitar así la delincuencia, la guerra, la cultura de masas degradada, las drogas, el aburrimiento y el ansia de poder, de distracción, de nirvana, el sexismo y el racismo. ${ }^{38}$

\footnotetext{
37 Fredric Jameson, Archaeologies of the Future: The Desire Called Utopia and Other Science Fictions (London: Verso, 2005), 147 y ss.

${ }^{38}$ Ibidem.
} 
El problema lo encontramos, entonces, cuando hablamos de las personas con enfermedades raras y de sus familias. Dijimos que estas personas están altamente condicionadas por sus enfermedades, tanto a nivel biomédico, como social. Recordemos que muchas enfermedades raras se manifiestan como síndromes y, además, un buen número de ellas, si no son mortales, tienen carácter crónico. En este sentido, son numerosos las consecuencias negativas asociadas a las enfermedades crónicas y que, de un modo u otro, terminan afectando a nivel socio-emocional a los afectados y a sus familias: angustia, ansiedad, aprensión a lo desconocido, intranquilidad, dolor, necesidad de períodos de hospitalización, cuidados diarios rutinarios, pérdida de autonomía, cambio en las relaciones familiares y sociales, perjuicio social, inseguridad e, incluso, temor a la muerte ${ }^{39}$. Ante esta realidad parece que solamente queda abierta la posibilidad para el chronos. Efectivamente, siempre es posible que las personas logren encontrar la oportunidad en elementos ajenos a estas limitaciones, pero, desde luego, las opciones son reducidas. De hecho, cuando se les pregunta a las familias y a las personas afectadas, es muy poco frecuente que hablen de lo que circunda a la enfermedad rara como un fenómeno abierto a la oportunidad. ${ }^{40}$ Todo este fenómeno de limitación del mundo como oportunidad encuentra su freno en la evolución de una sociedad más inclusiva y acogedora. De ahí que si mantenemos una sociedad centrada en ese proceso de homogeneización del que hemos hablado y en el que se produce, tal y como decía Sartori $^{41}$, un imperio de la mayoría, no habiendo opciones para grupos más reducidos con sus alternativas y dinámicas propias, será imposible que estas personas puedan llegar a percibir ese Kairós del que hemos hablado en su propio contexto. Estamos, entonces, ante

\footnotetext{
${ }^{39}$ Ana Belén Quesada, M ${ }^{\mathrm{a}}$ Dolores Justicia, Miriam Romero y M $\mathrm{M}^{\mathrm{a}}$ Trinidad García, "La enfermedad crónica infantil. Repercusiones emocionales en el paciente y en la familia", International Journal of Developmental and Educational Psychology INFAD Revista de Psicología 1, núm. 4 (2014): 569-576. http://dx.doi.org/10.17060/ijodaep.2014.n1.v4.832.

40 Juan R. Coca (coord.), Enfermedades raras. Contribuciones a la investigación social y biomédica (Soria: CEASGA, 2019); Juan R. Coca, Lidia Sanz-Molina, Hasier Eraña y Joaquín Castilla, "Análisis cualitativo del impacto social y familiar de las encefalopatías espongiformes transmisibles humanas", Revista de Neurología, 6, núm. 69 (2019): 242-249. https://doi.org/10.33588/rn.6906.2019122. Gema Esteban, Juan R. Coca, José Manuel Guerra de los Santos y Dianne Ruiz-Castañeda, "Análisis de la calidad de vida en personas con el Síndrome de Wolfram y de sus cuidadores: aspectos socio-psicológicos", OBETS. Revista de Ciencias Sociales 2, núm. 15 (2020): 471-500. https://doi.org/10.14198/OBETS2020.15.2.04.

${ }^{41}$ Giovanni Sartori, La sociedad multiétnica. Pluralismo, multiculturalismo y extranjeros (Madrid, Santillana, 2001).
} 
el imperio del chronos en el que solamente encontramos el tiempo, la celeridad, liquidez y evanescencia sin oportunidad transformativa.

Además, Roland Boer ${ }^{42}$ hace un análisis del concepto de Kairós y lo reactualiza de un modo interesante, especialmente útil para nuestro trabajo. Él indica que este concepto, en su perspectiva clásica, mantiene relación con el de milagro. Por lo tanto, y desde la perspectiva marxista que es la que él emplea, el Kairós tiene una fuerte vinculación con las élites de poder y con el capitalismo, puesto que el código teológico es inadecuado para la revolución. En este sentido, indica que existe una trampa relacionada con la posibilidad de realizar oposición a las clases dominantes. Ello es debido a que estos grupos sociales, como detentadores de poder, van a ser también los reguladores del mensaje teológico y, por ende, éste terminará siendo una forma de afirmar el derecho y el papel marginador de estas clases dominantes. Por ello, Boer defiende una perspectiva akairológica en la que cambie la concepción de la oportunidad y de la oposición transformadora.

Esta idea de la reducción en la perspectiva teológica del Kairós tiene interés social y político, puesto que las instituciones no tienen que atender a elementos teológicos. En este sentido, es importante ser conscientes que la realidad social necesita de estructuras sociales que atiendan a las necesidades sociales y les permita ampliar, en lo posible, las opciones sociosemióticas y sociohermenéuticas a colectivos tan vulnerables como el de las personas afectadas por enfermedades raras. Estas estructuras institucionalizadas requieren estar centradas en el fortalecimiento de la communitas, de los vínculos entre la comunidad y de la fraternidad. En este sentido, es importante romper con el imperio del chronos y ofrecer un tempo político y social a las personas afectadas. Un tempo para la oportunidad de ampliar su mundo-de-la-vida en sociedad y que, por esto mismo, necesita de cierta reducción del sentido teológico del Kairós. Ello no quiere decir que estemos rechazando las creencias religiosas como elementos kairológicos de interés para que las personas afectadas y sus familias tengan más opciones de sentido por estas vías. Lo que pretendemos afirmar, finalmente, es que las estructuras sociales necesitan ser conscientes de los diferentes aspectos a los que hemos hecho mención en este trabajo, para seguir 42 Roland Boer, "Revolution in the Event: The Problem of Kairós", Theory, Culture and Society 2, núm. 30
(2013): 116-134. 
conformando elementos que incrementen las posibilidades de sentido de las personas afectadas (directa o indirectamente) por las enfermedades raras.

Por todo ello, consideramos que es posible defender un tempo social y político alternativo. En él, la hermenéutica del tiempo podría ser analógica y adaptada a las necesidades que nos plantean este colectivo. Para ello sería necesario conformar instituciones o elementos institucionales donde los requisitos del chronos no estructuren su funcionamiento y opere como un frat tempore, un tiempo para la fraternidad. Queda, entonces, pendiente de futuros trabajos el desarrollo de este tiempo para la fraternidad. 


\section{Bibliografía}

Archer, Margaret S. Realist Social Theory: The Morphogenetic Approach. Cambridge: Cambridge University Press, 1995.

Archer, Margaret S. Culture and Agency: The Place of Culture in Social Theory. Cambridge: Cambridge University Press, 1996.

Archer, Margaret S. Being Human: The Problem of Agency. Cambridge: Cambridge University Press, 2009.

Band, Patrice F. "Vignette: Autoimmune Polyendocrine Syndrome Type I (APS 1)". En Rajeev K. Bali, Lodewijs Bos, Michael Christopher Gibbons y Simon Ibell (eds.), Rare Diseases in the Age of Health 2.0 (21-25). Berlin, Heidelberg: Springer-Verlag, 2014. DOI: https://dx.doi.org/10.1007/978-3-642-38643-5_2.

Bericat, Eduardo. Excluidos de la felicidad. La estratificación social del bienestar emocional en España. Madrid: CIS, 2018.

Berman, John. Enfermedades raras y medicamentos huérfanos. Claves para entender y tratar las enfermedades comunes. Barcelona: Elsevier, 2015.

Beuchot, Mauricio. Las caras del símbolo: el ícono y el ídolo. Madrid: Caparrós Editores, 1999.

Beuchot, Mauricio. Tratado de hermenéutica analógica. Hacia un nuevo modelo de interpretación. México: UNAM, 2015.

Beuchot, Mauricio. Hechos e interpretaciones. Hacia una hermenéutica analógica. México: Fondo de Cultura Económica, 2016.

Boer, Roland. "Revolution in the Event: The Problem of Kairós". Theory, Culture and Society 2, núm. 30 (2013): 116-134.

Bratman, Michael E. Structures of Agency: Essays. New York: Oxford University Press, 2007.

Bratman, Michael E. "Shared Agency”. En Chysostomos Mantzavinos (ed.), Philosophy of the Social Sciences. Philosophical Theory and Scientific Practice. Cambridge: Cambridge University Press, 2009.

Coca, Juan R. (coord.). Enfermedades raras. Contribuciones a la investigación social y biomédica. Soria: CEASGA, 2019. 
Coca, Juan R., Lidia Sanz-Molina, Hasier Eraña y Joaquín Castilla. “Análisis cualitativo del impacto social y familiar de las encefalopatías espongiformes transmisibles humanas". Revista de Neurología 6, núm. 69 (2019): 242-249. https://doi.org/10.33588/rn.6906.2019122.

Coca, Juan R., y Susana Gómez-Redondo. "Multidimensional Sociohermeneutics on Social Studies: a pragmatic and integrative perspective". En VV. AA., Approaches to the Concept of Trans-Subjectivity. Soria: CEASGA, 2020.

Crespo, Eduardo. "Un enfoque social sobre las emociones". En José Luis Álvaro (coord.), La interacción social. Escritos en homenaje a José Ramón Torregrosa. Madrid: CIS, 2018.

Dueñas, María, Begoña Ojeda, Alejandro Salazar, Juan Antonio Mico e Inmaculada Failde. "A Review of Chronic Pain Impact on Patients, Their Social Environment and the Health Care System". Journal of Pain Research núm. 9, (2016):457-467. https://doi.org/10.2147/JPR.S105892.

Esteban, Gema, Coca, Juan R., Guerra de los Santos, José Manuel y Ruiz-Castañeda, Dianne, "Análisis de la calidad de vida en personas con el Síndrome de Wolfram y de sus cuidadores: aspectos socio-psicológicos". OBETS. Revista de Ciencias Sociales 2, núm. 15 (2020): 471-500. https://doi.org/10.14198/OBETS2020.15.2.04.

Goffman, Erving. Estigma. La identidad deteriorada. Buenos Aires: Amorrortu, 2010.

Grills, Scott. "Understanding Everyday Life: Generic Social Processes and the Pursuit of Transcontextuality". Symbolic Interaction 4, núm. 43 (2019): 615-636.

Habermas, Jünger. Teoría de la acción comunicativa. Madrid, Trotta, 2018.

Haslam, Alexander, Charlotte McMahon, Tegan Cruwys, Catherine Haslam, Jolanda Jetten y Niklas Steffens. "Social cure, what social cure? The propensity to underestimate the importance of social factors for health". Social Science \& Medicine, núm. 198 (2018): 14-21. https://doi.org/10.1016/j.socscimed.2017.12.020.

Huete, Agustín, y Eduardo Díaz. Estudio sobre situación de Necesidades Sociosanitarias de las personas con Enfermedades Raras en España. Estudio ENSERio. Madrid: FEDER, 2009.

Jameson, Fredric. Archaeologies of the Future: The Desire Called Utopia and Other Science Fictions. London: Verso, 2005. 
Kjeldsen, Jens E. "Reconceptualizing Kairos". En Eyjólfur Kjalar Emilsson, Anastasia Maravela y Mathilde Skoie (eds), Paradeigmata: studies in honour of Øivind Andersen (249-258). Athens: The Norwegian Institute at Athens, 2014.

León, Emma. Sentido ajeno: competencias ontológicas y otredad. Barcelona: Anthropos, 2005.

Lévinas, Emmanuel. Fuera del sujeto. Madrid: Caparrós Editores, 1997.

Mead, George Herbert. Mind Self \& Society. The Definitive Edition. Edición de Charles W. Morris. Edición anotada de Daniel R. Huebner y Hans Joas. Chicago, London: The University of Chicago Press, 2015.

Muga, Jesús. El tiempo hebreo. Referentes antropológicos. Madrid: Fundación Emmanuel Mounier, 2002.

Niles, Paulomi Mimi, Saraswathi Vedam, Amy Witkoski Stimpfel y Allison Squires. "Kairos Care in a Chronos World: Midwifery Care as Model of Resistance and Accountability in Public Health Settings". Birth. Issues in Perinatal Care núm. 00 (2021): 1-13. https://doi.org/10.1111/birt.12565.

Quesada, Ana Belén, Ma. Dolores Justicia, Miriam Romero y Mª Trinidad García. "La enfermedad crónica infantil. Repercusiones emocionales en el paciente y en la familia". International Journal of Developmental and Educational Psychology INFAD Revista de Psicología 1, núm. 4, (2014): 569-576. http://dx.doi.org/10.17060/ijodaep.2014.n1.v4.832.

Richter, Trevor, Sandra Nestler-Parr, Robert Babela, Zeba M. Khan, Theresa Tesoro, Elizabeth Molsen, Dyfrig Hughes, et al. "Rare Disease Terminology and Definitions - A Systematic Global Review: Report of the ISPOR Rare Disease Special Interest Group" Value in Health 6, núm. 18 (2015): 906-914. https://doi.org/10.1016/j.jval.2015.05.008.

Sartori, Giovanni. La sociedad multiétnica. Pluralismo, multiculturalismo y extranjeros. Madrid: Santillana, 2001.

Von der Lippe, Charlotte, Plata S. Diesen y Kristin B. Feragen. "Living with a Rare Disorder: A Systematic Review of the Qualitative Literature". Molecular Genetics \& Genomic Medicine 6, núm. 5 (2017): 758-773. https://dx.doi.org/10.1002/mgg3.315. 